\title{
Design of UHF RFID Tag Antenna for Automotive Tire
}

\author{
Nadir Sarsri \\ Electronics and Telecommunication \\ Systems Research Unit \\ National School of Applied Sciences \\ (ENSA) Ibn Tofail University \\ Kenitra, Morocco \\ nadir.sarsri@uit.ac.ma
}

\author{
Sami Myllymäki \\ Microelectronics Research Unit Faculty \\ of Information Technology and Electrical \\ Engineering University of Oulu \\ Oulu, Finland \\ sami.myllymaki@oulu.fi
}

\author{
Marko Sonkki \\ Center for Wireless Communications \\ University of Oulu \\ Oulu, Finland \\ marko.sonkki@oulu.fi
}

\author{
Mohamed Nabil Srifi \\ Electronics and Telecommunication Systems Research Unit \\ National School of Applied Sciences (ENSA) Ibn Tofail University \\ Kenitra, Morocco \\ srifimn@ieee.org
}

\begin{abstract}
In this paper, a novel UHF RFID Tag antenna for automotive tire is presented. The antenna shape and size are optimized by using a meander line. To achieve a proper impedance matching between the antenna and the microchip a T-match is performed. The square-load is added at its ends to improve the bandwidth. The tag has a reduced size: $80 \mathrm{~mm} \times 12 \mathrm{~mm} \times 1.6 \mathrm{~mm}$. Hence, it can be attached simply in automotive tire. The tag provides the simple and low cost fabrication. The measured and simulated results show that the impedance matching is very convenient with broad impedance bandwidth. Moreover, the proposed antenna has omnidirectional radiation patterns and appreciable gain that satisfy maximum power transmitting in RFID communication.
\end{abstract}

Index Terms - antennas, radio frequency identification (RFID), tags, automotive tire.

\section{INTRODUCTION}

In recent years, radio frequency identification (RFID) has become very popular in many applications domains, such as purchasing and distribution logistics, manufacturing companies and material flow systems. That is provide wirelessly information about different items [1].

In RFID technology, the transponders "tags" can be classified in two categories: active (with power supply) or passive (no power supply) [2]. These latters communicate with the base station (reader) using an operating frequency. Each country adopts one special frequency band for RFID technology [3]. A typical passive RFID tag is composed of two main elements: an antenna and an application specific integrated circuit (ASIC) chip [4].

A passive RFID system operates in the following way (see Fig. 1). A reader antenna transmits a query signal, which is captured by the tag antenna. The radio frequency (RF) voltage established on tag antenna is converted to direct current [5]. This voltage activates the chip, and then the tag send data back by varying its input impedance. The impedance usually switches between two different states: conjugate match and

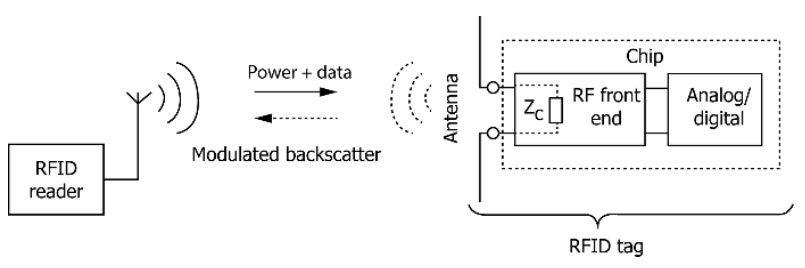

Fig. 1 RFID system operation

some other impedance values, successfully modulating the back-scattered signal [5].

The RFID system does not need line-of-sight to collect information about interrogated items [6]. Therefore, an RFID tag can be embedded into objects.

Generally, to develop a robust RFID system for automotive tire, there are two essentials challenges [6]. The first one is reliability and efficiency of the RF link between the tag and reader. The second one relates to structural persistence and durability of tag. Further, the tag antenna must have the following performances: (i) compact size to be attached to the required objects; (ii) omnidirectional radiation patterns; (iii) better impedance match; (iv) be robust to endure the mechanical effects caused by the tire while moving; (v) be cheap.

In passive RFID tag, the antenna should be self-resonant [7]. Therefore the matching process is designed with antenna to ensure low cost and maximum transmitted power between tags and reader. It is worth noting that ASIC chip has complex impedance, so ensuring proper impedance match is a challenging step.

In this work, we present a UHF RFID tag antenna for automotive tire. The size reduction technique is performed by using a meander line, whereas impedance matching is simply achieved by a T-match method. Besides, antenna shape and used materials are appropriate for low cost fabrication. The section II discusses the design methodology of the proposed 


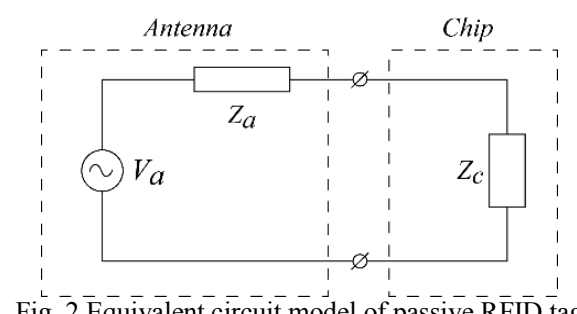

Fig. 2 Equivalent circuit model of passive $\bar{R} \bar{F} \overline{\mathrm{D}} \overline{\mathrm{t}}$ tag

antenna, while section III presents the results and the performances of the proposed antenna. Finally, section IV offers a brief conclusion.

\section{TAG ANETNNA DESIGN}

\section{A. Impedance Matching}

A typical passive tag consists of an antenna and an ASIC chip. Both exhibit the respective complex impedance $Z_{a}=R_{a}$ $+j X_{a}$ and $Z_{c}=R_{c}+j X_{c}$, where $Z_{a}$ is the input impedance of the antenna and $Z_{c}$ is the input impedance of the tag [8]. More precisely, its equivalent circuit model can be simply described using Thevenin model as shown in Fig. 2. The chip can use the entire power available at antenna if a perfect impedance matching is performed [9]. Therefore, the impedance antenna must be conjugate of the chip one.

The complex power reflection coefficient $\Gamma$ can be defined as:

$$
\Gamma=\frac{z_{c}-z_{a}^{*}}{z_{c}+z_{a}}
$$

Then the power reflection coefficient can be calculated by:

$$
|\Gamma|^{2}=\left|\frac{z_{c}-z_{a}^{*}}{z_{c}+z_{a}}\right|^{2}
$$

So to deliver the maximum power available at the antenna (considered as source) to the chip (considered as load), the power reflection coefficient should be null.

More specifically, the power transmission coefficient, $\tau$, can be defined as:

$$
P_{c}=\tau P_{a}
$$

Where $P_{a}$ stands for the power collected by the tag antenna and $P_{c}$ refers to the power at the chip. We can also express the power transmission coefficient $\tau$ by:

$$
\tau=1-|\Gamma|^{2}
$$

By using (2) and (4), the power transmission coefficient can be expressed in terms of impedances by:

$$
\tau=\frac{4 R_{a} R_{c}}{\left|Z_{c}+Z_{a}\right|^{2}}
$$

In general, the ASIC chip used in RFID tag has an important negative imaginary part and small real part [10]. We can admit that the chip has capacitive behavior in order to store energy. Hence, the designed antenna should have inductive behavior in order to minimize the reflection coefficient $\Gamma$ and maximize the quantity of power delivered to the ASIC chip.

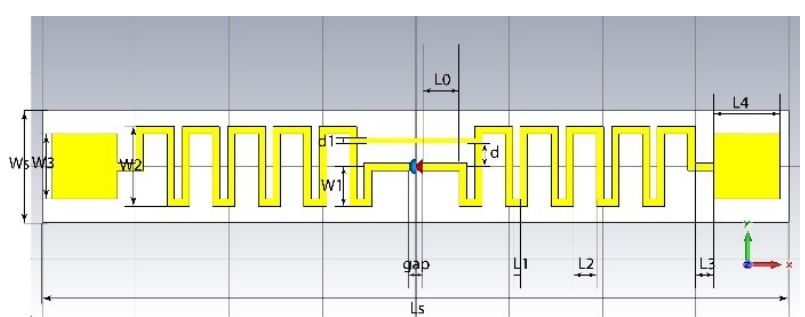

Fig. 3 Geometry of the proposed tag antenna

\section{B. Read Range}

To wake up the tag, in most RFID scenarios, the reader interrogates the tag by sending query signal. Hence, the RFID tag should be present in the vicinity. Now, we can talk about read range. This latter is the maximum distance at which RFID tag can notice a minimum power from reader signal and the tag can deliver necessary information to the base station [9]. Therefore, we can consider two distances one in uplink and another in downlink. The read range is the smaller of these two distances because tag sensitivity is generally low [11]. The read range depends also on propagation conditions, tag antenna polarization and material of tagged Item [11]

By using Friis formula, the read range $r$ can be calculated as:

$$
r=\frac{\lambda}{4 \pi} \sqrt{\frac{P_{t} G_{t} G_{r} \tau}{P_{t h}}}
$$

Where $\lambda$ is the wavelength, $P_{t}$ is the power transmitted by the reader, $G_{t}$ is the gain of the reader antenna (transmitter), $G_{r}$ is the gain of the tag antenna (receiver), $P_{t h}$ is the minimum threshold power necessary to turn on the RFID tag chip and $\tau$ is the power transmission coefficient.

We have to note that the impedance match condition $(\Gamma=0)$ affects the performances of tag antenna, particularly the read range. With a view to increasing the read range, the impedance matching should be perfectly performed.

A set of requirements must be taken into account while designing an RFID tag antenna. The designer can deal between antenna gain, impedance matching, and bandwidth [11].

\section{Tag Antenna Design}

Our design approach is based on recommendations descripted on [11]. The tag has been designed to be attached to automotive tire in order to collect their inventory information and conditions (pressure, temperature, stress, road conditions etc). Furthermore, sensors may be included with tags. Then the tag can communicate with reader within an operation frequency.

We opted for the 902-928 MHz band. We selected the 915 $\mathrm{MHz}$ as operating frequency for our tag antenna. To reduce the cost and have a straightforward PCB integration, it has been decided to use the FR4 as antenna substrate. Its dielectric permittivity and thickness are respectively $\varepsilon_{r}=3.5, \mathrm{t}_{\mathrm{s}}=1.6 \mathrm{~mm}$. The antenna trace is made of copper with a thickness $\mathrm{t}_{\mathrm{a}}=0.018$ $\mathrm{mm}$ and trace width $W=0.8 \mathrm{~mm}$. 


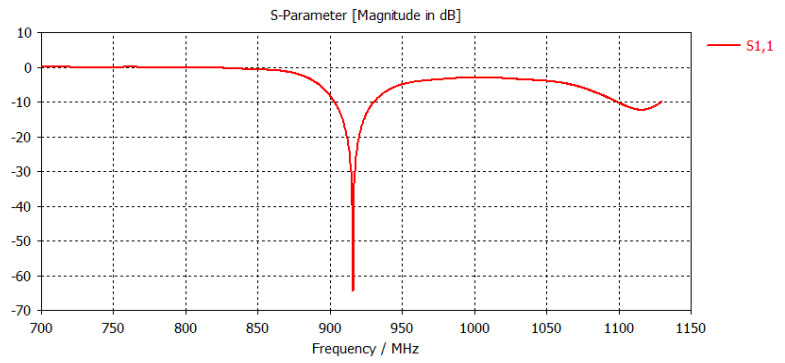

Fig. 4 Simulated reflection coefficient

For our application, we chose the NXP UCODE G2XL chip [12] with TSSOP8 packaging. The integrated chip (IC) exhibits a complex impedance $Z_{c}=16-j 148$ at $915 \mathrm{MHz}$ frequency. It has a low resistance part and a relatively high reactance part.

The Fig. 3 shows the shape and structure of our proposed RFID tag antenna. In order to reduce the antenna size, meandered line antenna (MLA) was performed. Meandering allowed the antenna to be compact and to provide omnidirectional performance in the plane perpendicular to the meander line [11]. As shown in Fig.3, the antenna is designed with a nonuniform MLA (NU-MLA) to increase the gain [7].

To perform the impedance matching, the well known Tmatch technique [10] was an intuitive choice. Most of passive RFID tag antenna suffers from narrowed bandwidth. So to deal with this problem, we decided to add a square patch at the ends of the meander line. Hence, we can talk about end-loaded antenna [13].

The proposed antenna geometry is optimized to be directly connected with the NXP UCODE G2XL chip. The tag antenna should exhibit the input impedance $Z_{a}=16+j 148$ at resonant frequency. In this case, tag performances will be improved.

\section{RESULTS AND DISCUSSION}

\section{A. Simulated Performances}

Design optimization was performed using CST Microwaves. Table I gives the optimized dimensions for the proposed tag antenna.

The simulated reflection coefficient is plotted in Fig. 4. The magnitude of the reflection coefficient is below $-64.26 \mathrm{~dB}$ at the desired frequency $915 \mathrm{MHz}$. We can affirm that the integrated chip receive the maximum power available at the proposed tag antenna. Therefore, the antenna impedance response is the perfect conjugate for the ASIC impedance. It is clear that we ensure excellent impedance match with chip. Having proper impedance matching could ensure a longer antenna reading distance, which is a paramount important requirement for RFID application.

Usually for RFID system the impedance bandwidth is calculated for $S_{11} \leq-10 \mathrm{~dB}$. For our case, the simulated impedance bandwidth is $27.2 \mathrm{MHz}$. It covers the allowed bandwidth in North America. The most RFID tag antenna suffer from narrowed bandwidth [7]. Hence, the proposed antenna has significant advantage in bandwidth aspect.
TABLE I. OPTIMIZED Dimensions OF PROPOSED ANTENNA IN MM

\begin{tabular}{|c|c|c|c|c|c|}
\hline Dimension & Value & Dimension & Value & Dimension & Value \\
\hline Ws & 12 & L0 & 4 & L3 & 2 \\
\hline W1 & 4.3 & Ls & 80 & L4 & 7 \\
\hline W2 & 8.6 & L1 & 0.8 & d & 2.5 \\
\hline W3 & 7 & L2 & 2.5 & d1 & 0.5 \\
\hline
\end{tabular}

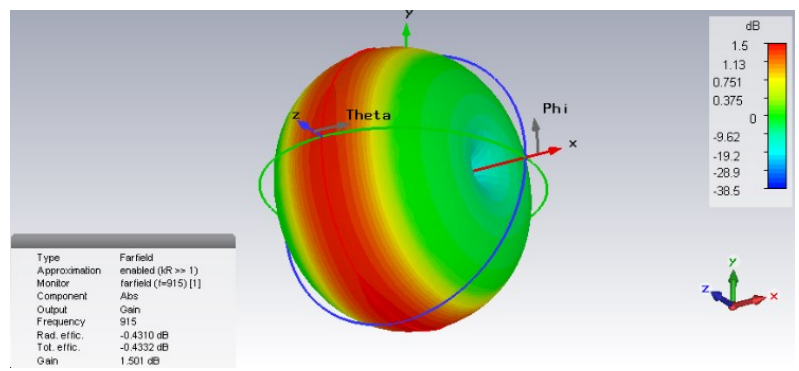

Fig. 5 Simulated 3D-Gain

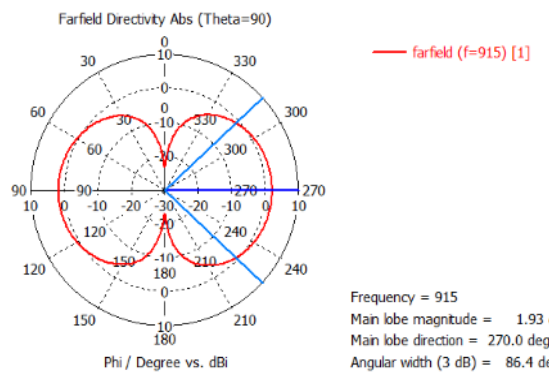

Fig.6 Two dimension 2D-directivity

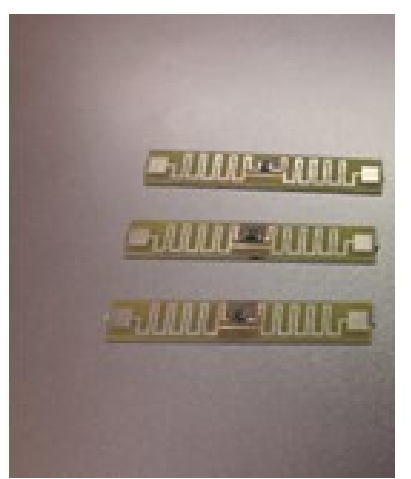

a)

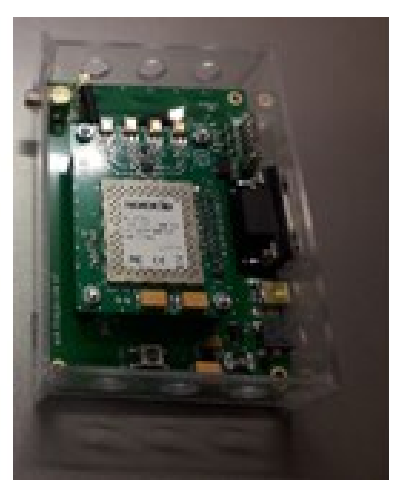

b)
Fig. 7: a) Photograph of the fabricated tags prototypes

b) Reader electronics made by NORDICID

Fig. 5 illustrates the simulated gain. The proposed tag antenna exhibits a maximum gain over the perpendicular plane to the antenna trace. As shown in Fig. 5, the maximum obtained gain is $1.5 \mathrm{~dB}$ at operation frequency. Compared to uniform MLA studied in [7], our tag antenna can provide considerable result in gain characteristic.

From the radiation patterns, the proposed antenna exhibits omnidirectional radiation. As depicted in Fig. 6, its maximum directivity is $1.93 \mathrm{~dB}$ at desired frequency when theta $=\mathrm{phi}=90^{\circ}$. Due to its omnidirectional radiation 


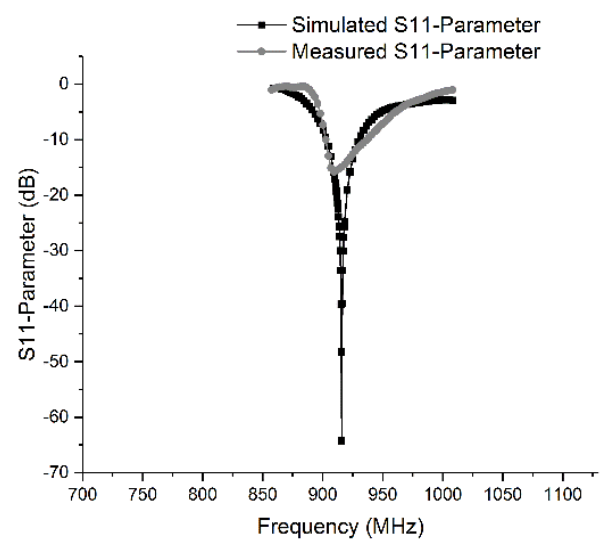

Fig. 8 Measured and Simulation S11 Parameter

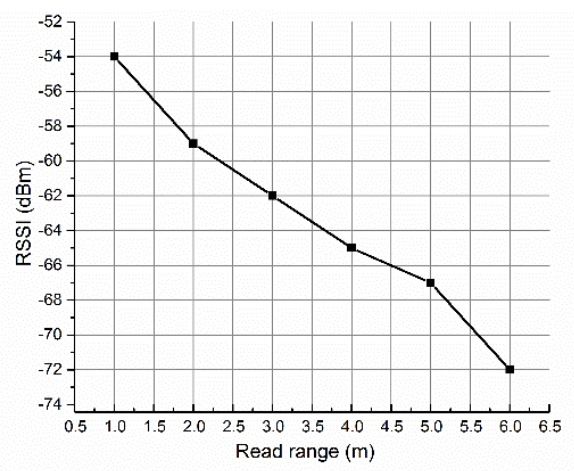

Fig 9. Measured Read range in meter

characteristic, the tag can be recognized by RFID readers even if it is placed in any position and angle.

\section{B. Experimental Results}

The proposed RFID Tag antenna was fabricated and tested (Fig. 7). A $900 \mathrm{MHz}$ monopole antenna was used to measure the tag's response. Fig. 8 shows the measured and simulated reflection coefficient parameter S11. Good agreement is obtained between measured and simulated results.

The tag read range was measured in free space by using a Wideband Dual-Polarized Cross-Shaped Vivaldi Antenna [14]. The transmitter has the following characteristics: $P_{t}=1000 \mathrm{~mW}$ and $G_{t}=4 \mathrm{dBi}(\mathrm{EIRP}=34 \mathrm{dBm})$. The calculated read range is based on received signal strength indicator (RSSI) that is backscattered by the tag and measured by reader antenna. The measured RSSI for different distances is illustrated in Fig. 9. The maximum read distance reaches 6 meters while maintaining acceptable RSSI value.

\section{CONCLUSION}

A novel passive RFID tag antenna for automotive tire was designed. The simulation and experimental results prove that our proposed antenna gives good performances for automotive tire application and it can be a good candidate for RFID system. The meander line was better solution to reduce the size. We have to note that the T-match technique ensures proper impedance matching. That is useful to increase read distance. The end-loading square patch improves the impedance bandwidth and radiation.

\section{ACKNOWLEDGMENT}

Authors would like to thank Professor Jantunen Heli and all Microelectronics research unit team and the Nordic ID Company for the help on antenna design and measurements. Author acknowledge the financial support of the erasmus + pragramme, Key 1 - Mobility for learners and staff - for the cooperation between Microelectronics research unit, University of Oulu, Finland, and Ibn Tofail University, Electronics and Telecommunication systems research unit, Morocco.

\section{REFERENCES}

[1] K. Finkenzeller, "RFID Handbook: Fundamentals and Applications in Contactless Smart Cards, Radio Frequency Identification and nearField Communication," $3^{\text {th }}$ Ed, Wiley 2010.

[2] S. Preradovic, N. Karmakar, I. Balbin, "RFID Transponders," IEEE Microwave Magazine, vol. 9, no. 5, pp. 90-103, Oct. 2008.

[3] V. Chawla, D. Sam Ha, "An Overview of Passive RFID," IEEE Communications Magazine, vol. 45, no. 9, pp. 11-17, Sept. 2007.

[4] K. Domdouzis, B. Kumar, C. Anumba, "Radio-Frequency Identification (RFID) Application: A brief introduction," Elsevier Advanced Engineering Informatics, vol. 21, no. 4, pp. 350-355, Oct 2007.

[5] K. V. S. Rao, "An Overview of backscattered radio frequency identification system (RFID)," Asia Pacific Microwave Conference, pp. 746-749, 30 Nov.- 3 Dec. 1999.

[6] S. Shao, L. Z. Lee, R. J. Burkholder, J. L. Volakis, "Embedded UHF RFID Tag Antennas for Automotive Tire Sensing" Antennas and Propagation Society International Symposium, pp. 1494-1495 2013 IEEE.

[7] G. Marrocco, "Gain-Optimized Self-Resonant Meander Line Antennas for RFID Applications," IEEE Antennas and Wireless Propagation Letters, vol. 2, no.3, pp. 302-305. 2003.

[8] K.V. S. Rao, P. V. Nikitin, S. F. Lam, "Impedance matching concepts in RFID transponder design" The Fourth IEEE Workshop on Automatic Identification Advanced Technologies, pp. 39-42, Oct. 17$18,2005$.

[9] P. V. Nikitin, K. V. Rao, S. F. Lam, V. Pillai, R. Martinez, H. Heinrich, "Power Reflection Coefficient Analysis for Complex Impedances in RFID Tag Design," IEEE Transactions on Microwave Theory and Techniques, vol. 53, no. 9, pp. 2721-2725 Sept. 2005.

[10] G. Marrocco, "The art of UHF RFID antenna design: impedancematching and size-reduction techniques," IEEE Antennas and Propag. Mag., vol.50, no.1, pp.66-79, Feb. 2008.

[11] K. V. S. Rao, P. V. Nikitin, S.F. Lam, “Antenna design for UHF RFID tags: a review and a practical application," IEEE Transactions on Antenna and Propagation, vol. 53,no. 12, pp. 3870-3876, Dec. 2005.

[12] http://www.nxp.com/documents/data_sheet/SL3ICS1002_1202.pdf

[13] S. Shao, A. Kiourti, R. Burkholder, L.Volakis, "Broadband TextileBased Passive UHF RFID Tag Antenna for Elastic Material," IEEE Antennas and Wireless Propagation Letters, vol.14, pp.1385-1388, Feb. 2015 .

[14] S. Marko et al, "Wideband Dual-Polarized Cross-Shaped Vivaldi Antenna," IEEE Transactions on Antennas and Propagation, Vol. 63, No. 6, Jun. 2015, pp. 2813 - 2819. 